\title{
La percolación cultural o cómo el mercado se institucionaliza en el tercer sector
}

\author{
José A. López Rey
}

Universidade da Coruña

Departamento de Socioloxía, Ciencia Política e da Administración

Campus de Elviña, s/n. 15071 A Coruña

jlopezr@udc.es

\section{Resumen}

Proponemos el concepto de percolación cultural para describir y estudiar el fenómeno de la introducción en el tercer sector de los valores propios de las organizaciones del mercado. Para ello, se describe un marco teórico que incluye teorías sobre la cultura organizacional, el nuevo institucionalismo y la teoría relacional de la sociedad como mejor herramienta para conocer el tercer sector. Se explicitan las hipótesis que han guiado la investigación y la metodología que la hizo posible y se refieren los principales hallazgos. En el nivel macro, se observa la percolación a través de dos tipos de comportamiento organizacional diferenciado; en el plano micro, se atiende a los factores ideológicos que dificultan la percolación; en el meso, se relaciona la percolación cultural con factores de naturaleza material. El análisis se realizó a partir de datos secundarios de 87 ONGD, 28 entrevistas semiestructuradas y una encuesta realizada al personal de 24 organizaciones.

Palabras clave: percolación cultural, valores de mercado, tercer sector, ONG, España.

\section{Abstract. Cultural percolation: business-like mentality appears into the Third Sector like institution}

In this article the concept of cultural percolation is used to describe and study the introduction of values of for-profit organizations in the Third Sector. We propose a theoretical frame that includes theories on organizacional culture, the relational theory of society and the new institutionalism. This article specifies several hypotheses and the methodology to verify them, and presents the results of the research carried out at three levels: First, at macro level, the cultural percolation is observed in two types of organizational behavior; second, at micro level, ideological factors which obstruct the cultural percolation are described; and third, at meso level, the cultural percolation are influenced by material factors. The analysis is based on secundary data of 87 Developmental Non Governmental Organizations and primary analysis of data from 28 semiestructurated intervews and a survey to the personnel of 24 organziations.

Key words: cultural percolation, values of for-profit, business-like mentality, Third Sector, NGO, Spain. 


\section{Sumario}

Introducción Resultados de la investigación

Fundamentos teóricos de la investigación Conclusiones

Hipótesis

Referecias bibliográficas

Metodología

\section{Introducción}

Las organizaciones no gubernamentales para el desarrollo (ONGD) se han convertido en un referente ético y político para la ciudadanía, pues este tipo de organizaciones «recobran la ilusión de que es posible poner en vigor la solidaridad y la hermandad en medio de un mundo desilusionado, desencantado y peligroso» (Giner, 1995: 21). Hay quien va más allá y afirma que son "el factor movilizador proactivo más vigoroso en la política informacional» (Castells, 1998: 390-391). Sin embargo, tras un primer momento en el que este tipo de organizaciones gozaron de la confianza casi total de los medios de comunicación, en cada vez más ocasiones se plantean dudas acerca de su comportamiento. Desde el ámbito periodístico (Cernuda, 2002), pero sobre todo académico (Sogge, 1998; Serrano, 2002; Sampedro, Jerez y López Rey, 2002; entre otros), se llama la atención sobre comportamientos que no coinciden con lo que se espera de una entidad de este tipo. Éste es el objeto de estudio del presente trabajo. La lógica o racionalidad propia de las organizaciones solidarias.

Cabría pensar que en las ONGD, como en cualquier organización, hay ciertos niveles de picaresca y de corrupción. Siendo esto cierto, son muchos y de muy diverso tipo los ejemplos existentes como para atribuirlos a la falta de honradez de algunos gestores de ONG, demasiado complejos como para entender que se trata solamente de prácticas fraudulentas o corruptas. En efecto, ante una ONG que se dedica a poner en marcha programas de salud en el Tercer Mundo y que recibe fondos de una empresa que crea enfermos con sus productos (como una tabacalera), no nos hallamos con un caso de corrupción, ni de fraude, sino con un contrasentido. Con algo que contradice la lógica con la que se supone trabajan, o han de trabajar, las organizaciones solidarias.

¿O no existe tal contradicción? La necesidad de obtener fondos y la complejidad creciente del entorno en el que operan las ONG demanda cada vez más trabajadores cualificados en lugar de voluntarios que presten su tiempo libre; cada año que pasa, es mayor la presencia de muchas ONG en los medios de comunicación como anunciantes y aumentan también las relaciones que establecen con empresas para obtener financiación para sus proyectos. Estos hechos, entre otros, hacen pensar que la lógica propia de las ONG se cuestiona o, como veremos aquí, entra en competencia con otras lógicas diferentes. 
Nosotros mantenemos que existe una penetración (o, como veremos más adelante, una percolación) cultural por parte del mercado en el seno del tercer sector que entra en conflicto con la mentalidad tradicional que existe en las organizaciones solidarias, en concreto, en las llamadas de cooperación internacional u ONGD (organizaciones no gubernamentales para el desarrollo). A continuación, desarrollaremos, de manera harto somera, el marco teórico de la investigación, sus hipótesis y metodología y sus conclusiones. También se expondrán unas consideraciones básicas de cara a futuros estudios.

\section{Fundamentos teóricos de la investigación}

\section{La cultura organizacional}

Dentro de las distintas escuelas que estudian la cultura organizacional, nos situamos en la perspectiva simbólica, que entiende la cultura como un producto de la mente que se erige en un sistema de significados y de símbolos colectivos, según el cual los individuos interpretan sus experiencias y orientan sus acciones. Se pueden ubicar en esta perspectiva comprensiva y constructivista, tanto la sociología interpretativa accionalista, como la corriente institucionalista y la derivada de la fenomenología, la etnometodología y el interaccionismo simbólico (Allaire y Firsirotu, 1992).

La cultura organizacional es «un modelo de presunciones básicas - inventadas, descubiertas o desarrolladas por un grupo dado al ir aprendiendo a enfrentarse con sus problemas de adaptación externa y de integración interna- que hayan ejercido la suficiente influencia como para ser consideradas válidas y, en consecuencia, ser enseñadas a los nuevos miembros como el modo correcto de percibir, pensar y sentir esos problemas» (Schein, 1988: 25-26). La cultura de la organización ofrece un sentido de identidad a los miembros, facilita la generación de compromisos, ya sean supraindividuales o de grupo, dota de estabilidad al sistema social abierto que es la organización y confiere sentido y significado a las conductas que acontecen en el seno de la organización (Smircich, 1983: 345-346).

Esta perspectiva diferencia entre dos planos, el socioestructural y el sociocultural, que están relacionados pero no son lo mismo, y contempla el cambio del sistema a partir de la interacción entre ambos, pero también de los elementos del entorno y de los propios miembros. Resulta especialmente adecuada esta perspectiva de la cultura organizacional, porque acepta la posible existencia de diversas culturas en la organización (Etkin y Schwarstein, 1989) que puedan llegar a competir entre sí (Riley, 1983). Esto nos conduce directamente al segundo apoyo teórico del que nos servimos en esta investigación. Se han estudiado ampliamente las creencias, las normas y los valores que tienen los empleados de las empresas y de las administraciones públicas. Pero ¿existen unas creencias básicas y compartidas entre los miembros de las ONG? ¿Qué es lo culturalmente característico del tercer sector en el que operan? 


\section{El tercer sector}

La literatura académica sobre el tercer sector se puede resumir en tres grandes perspectivas (Herrera Gómez, 1998). Hay teorías económicas (Lebesque, 1989; Desroche, 1983; Hansmann, 1987; Krashinsky, 1986; Weisbrod, 1998; Douglas, 1983; Sjöstrand, 2000, entre otros) y las hay de corte sociológicoadministrativo (Salamon, 1987a, 1987b y 1999; Salamon y Anheier, 1992a, 1992 b y 1998). Todas ellas se han preocupado del estudio del tercer sector teniendo como base los paradigmas utilitaristas, racionalistas e individualistas (teoría de juegos, de la elección racional, de elección pública, del obrar administrativo, etc.) y, a grandes rasgos, se les pueden achacar dos carencias importantes: no son capaces de explicar el hecho solidario en sí mismo y, por lo tanto, no pueden dar cuenta del tercer sector sin referenciarlo al espacio público estatal ni al privado del mercado (normalmente subordinándolo a éstos). Con matices, esta misma crítica se le puede hacer a la mayor parte de las teorías de corte sociológico-cultural (Herrera Gómez, 1998). No obstante, existe una tercera perspectiva, la relacional de la sociedad, que se muestra capaz de explicar el tercer sector y los procesos que ocurren en él. Y lo hace partiendo de tradiciones teóricas que entroncan directamente con Durkheim y Parsons (Herrera Gómez, 1998; Donati, 1978, 1991 y 1997).

Donati (1997) parte de la premisa de que ese espacio siempre ha existido, incluso antes de que el mercado y el estado hicieran su aparición como productos sociales característicos de la modernidad. El elemento específico del tercer sector sería la propia relacionalidad social y, en concreto, la acción altruista, gratuita o solidaria. Se entiende que la relacionalidad social, antes de que expresara valores de intercambio (en el mercado) y de que fuera objeto de regulación política y jurídica (por parte del estado), ya se expresaba en un espacio propio que, en la medida en que la sociedad se hace más compleja y estructuralmente diferenciada, se ha ido especificando en un sector con instituciones sociales propias: el tercer sector y las ONG.

Sin profundizar en este momento en esta teoría, sí podemos decir que Donati aplica el sistema AGIL a la solidaridad para evidenciar sus aspectos relacionales y para comprender cómo se diferencia en las sociedades modernas. De igual modo, introduce la solidaridad entre los medios de intercambio sociales como el dinero, el poder o los afectos, de forma que el tercer sector puede ser explicado en un plano de igualdad con los otros. El tercer sector tendría, entonces, una serie de características generales, de las que resaltamos ahora tres:

- Una cultura propia basada en el altruismo, el don, la reciprocidad, la solidaridad o la confianza.

- Una normatividad característica.

- Un modo de funcionamiento que expresa formas organizativas caracterizadas por el hecho de movilizar recursos peculiares y de combinarlos según modos propios, como, por ejemplo, las ONG (Donati, 1997), pero también los movimientos sociales. 
Ahora bien, el análisis de la literatura académica sobre la cultura organizacional del tercer sector nos informa de que existen una serie de hechos (formas de reclutamiento del personal, tipos de gerencia más profesionalizados y sobre todo determinadas maneras de captar fondos) que informan de actuaciones propias del mercado en el tercer sector y que evidencian la competencia cultural entre ambos. Se hace imprescindible, entonces, atender a cómo es esta competencia, lo que nos lleva al tercer pilar teórico: la teoría neoinstitucionalista.

\section{La institucionalización de la solidaridad en España}

Resulta pertinente la idea de institución. Una definición que nos acerca al significado de este concepto es la de Douglass North, quien afirma que las instituciones son las reglas del juego en una sociedad o, más formalmente, los constreñimientos u obligaciones creados por las personas que dan forma a la interacción humana (North, 1990).

Y en nuestra cultura está fuertemente arraigada la idea de que los actos solidarios, ya sean de tipo filantrópico individual u organizado, deben estar guiados por un espíritu muy diferente al que regula la actividad comercial. En efecto, la institucionalización de la solidaridad en España se ha producido de manera diferente a la de otros entornos culturales próximos (como en los EEUU o en Gran Bretaña) donde el Charity Sector tiene un fuerte componente mercantil. En nuestro país, la práctica de la solidaridad partió de presupuestos ideológicos distintos al de la racionalidad económica de mercado. Las explicaciones a este hecho son múltiples y variadas, pero cabe señalar el papel destacado que tuvieron los movimientos socialista y anarquista y, sobre todo, el de la Iglesia católica (Orella, 2000: 48), que erigieron marcos compartidos de modelos mentales contrarios a la lógica capitalista de mercado. Y, bajo estos constreñimientos culturales, se ha socializado gran parte de la sociedad española. En otras palabras, y refiriéndonos a la relación existente actualmente entre el tercer sector y el mercado, a la solidaridad no se le pedía que fuera eficaz ni eficiente, no se exigía a las ONGD la gestión estratégica, la necesidad de una formación de postgrado para directivos, la presencia en los medios de comunicación, el gasto en publicidad, sino simplemente que sus acciones fueran realmente solidarias. Pero ahora sí, nos dicen desde muchas ONGD, el entorno demanda los elementos antes citados para seguir existiendo. Esto sugiere la idea de un cambio institucional, una modificación de la cultura de las organizaciones en la que se incorporan nuevas creencias, paradigmas, códigos y conocimientos que antes no existían. En definitiva, los que imperan en el mercado. Pero este cambio de una forma de entender la solidaridad organizada a otra resulta sumamente difícil, habida cuenta de que son los propios acuerdos institucionales los que descartan determinadas líneas de acción definiéndolas como inviables.

Existen, no obstante, determinadas situaciones en las que el cambio institucional es posible. Una de ellas es la transformación en el marco de la rela- 
ción de las organizaciones que operan en el mismo entorno (North, 1990). En principio, es difícil que una institución dé paso a otra. Si esto ocurre, la nueva institución ha de estar más en consonancia con el entorno y, sobre todo, más legitimada por éste. Mary Douglas (1986: 52) ha desarrollado un argumento según el cual la legitimidad de las instituciones depende de su capacidad para sostener analogías naturalizadoras, es decir, para aparecer como algo natural y, por tanto, no una creación humana. En este sentido, hay una perspectiva crítica que define la economía capitalista como el orden institucional dominante. Uno de los autores más representativos de esta línea de análisis, Claus Offe, argumenta que el capitalismo genera la dinámica del sistema social y, no obstante, «neutraliza el significado» del que cada vez más depende su reproducción (Offe, 1984: 82). En el fondo, lo que ocurre es que la economía capitalista se convierte no ya en el orden institucional dominante, sino en el hegemónico, habida cuenta de que utiliza la cultura como elemento de conservación del orden social capitalista institucionalizándose en los diferentes espacios sociales a través de ella (Habermas, 1987).

\section{Hipótesis}

Nuestra hipótesis es que se ha producido una percolación cultural por parte del mercado en las organizaciones del tercer sector, en concreto, en las ONGD. Habida cuenta de la novedad del concepto, debemos justificar su adopción frente a otros más habituales. El de percolación es un concepto preciso; se refiere a un proceso y, como tal, establece sus causas, sus efectos y asume que existen factores resistentes a ese proceso. Aunque proviene de las ciencias naturales, tiene un uso creciente en las ciencias sociales, y en todas sus acepciones mantiene una unidad semántica.

En agronomía, significa «la filtración de líquidos en el suelo, por acción de la fuerza de la gravedad, hasta las capas profundas de la tierra» (Decker y Frey, 1996); en esta disciplina, se contempla la escorrentía junto a la percolación, esto es, los daños causados por el exceso de humedad. En física, la percolación describe las transiciones de fase crítica que ocurren en los sistemas complejos. Por ejemplo, en una red formada por diversos elementos, existe un umbral o probabilidad crítica a partir del cual uno de los elementos se expande por el sistema; el proceso es la percolación y el umbral crítico, su coeficiente (Albert y Barabási, 2002). El fenómeno de la percolación ocurre en las redes complejas, sea cual sea su naturaleza, motivo por el cual puede darse también en las redes sociales (Watts, 2003). En este campo, la percolación se refiere a las posibilidades de difusión de un conjunto de ideas en una población que se caracteriza por diversos umbrales de resistencia al cambio.

Por todo esto, el concepto de percolación cultural resulta no sólo adecuado, sino también idóneo. En ciencias sociales, significa la extensión (o contagio) de una idea, un comportamiento o una moda (Watts, 2003; Miceli, 2006) y estaría relacionado con el concepto de isomorfismo institucional. En el caso que nos ocupa, la percolación cultural sería el fenómeno por el cual la cultura 
empresarial, o los modos y las lógicas institucionales que caracterizan a las organizaciones del mercado se estarían extendiendo o percolando por las ONGD, desplazando a la lógica con la que éstas tradicionalmente vendrían operando (no sólo en materia de gestión de la imagen o gestión de la organización, sino también en lo referido al lenguaje y, en general, todo aquello relacionado con la cultura organizacional).

La lógica tradicional de las ONGD, la que caracterizaba al tercer sector frente a la del mercado (maximización del beneficio) o a la del estado (la lógica de la burocracia, la aplicación de la norma), es la del don, la del altruismo, la de la generosidad, en definitiva, la solidaridad de suyo. Es, por tanto, una dimensión puramente expresiva y en la que no intervienen (no al menos necesariamente) cálculos para optimizar recursos y maximizar beneficios, puesto que lo más importante no es la acción en sí, sino el sentido con el que se actúa. Sin embargo, la acción de una ONGD guiada por una lógica percolada desde el mercado tiene en cuenta la optimización de recursos y el análisis de impacto económico y mediático, de manera que la acción pueda ser rentabilizada en términos de imagen y capital simbólico para que, a su vez, redunden en el futuro en un incremento de los ingresos.

Creemos que la idoneidad del concepto de percolación cultural se basa en dos aspectos. Por un lado, porque se refiere de manera precisa cómo ocurre el fenómeno. En efecto, describe cómo hay un punto a partir del cual la cultura que tradicionalmente ha caracterizado a las organizaciones del tercer sector comienza a verse desplazada, alterada o, por seguir con la metáfora, erosionada por causa de la nueva lógica institucional. Y este momento debe poder concretarse en el tiempo. Pero, además, la percolación hace referencia también no sólo al cómo, sino también a por qué ocurre el fenómeno. De la misma manera que la filtración de agua ocurre por la fuerza de la gravedad, el fenómeno social es «inevitable» por cuanto la economía capitalista es el orden social dominante que hace de la cultura un elemento funcional en la conservación del sistema. En otras palabras, la lógica mercantil tiende a ocupar espacios institucionales que no le son propios (como la familia, el estado o, en el caso que nos ocupa, el tercer sector) para reforzar la legitimidad del capitalismo.

La hipótesis de la percolación se desglosa en otras subhipótesis operativas relacionadas con los fundamentos teóricos vistos anteriormente:

- Primera: proponemos la existencia de dos racionalidades diferentes en las ONGD: una, que definimos como la solidaridad expresiva, se correspondería con la que Donati define como medio de intercambio simbólico generalizado propio del tercer sector. La otra, que llamamos economicista o percolada, va más allá de la instrumentalidad que cabe esperar que exista en cualquier organización y refleja la introducción de los valores propios del mercado.

- Segunda: creemos que, en la historia reciente de las ONGD en España, se ha producido un cambio en el entorno organizacional que ha modificado sustancialmente el entramado institucional, lo que ha permitido el conflicto entre las dos lógicas ya señaladas. 
- Tercera: creemos que, dentro del sector organizacional, se pueden encontrar pautas de comportamiento diferenciadas en función de diversas variables del plano socioestructural, y relacionadas con las lógicas expresiva y economicista.

- Cuarta: finalmente, los elementos más resistentes a la adopción de la lógica percolada desde el mercado serán aquellos individuos con un mayor grado de ideologización en materia de creencias religiosas y/o con la ideología política de izquierdas.

\section{Metodología}

Debido a los diferentes planos a los que se refiere esta investigación, se hizo necesario adoptar un planteamiento metodológico pluralista que asumiera naturalmente la integración macro-micro y que permitiera la utilización de herramientas adecuadas de tipo cuantitativo y cualitativo. Se trabajó en dos planos. Primero, el conjunto de ONGD que conforman la Coordinadora estatal (CONGDE), 87 organizaciones, que definimos como el universo organizacional. Además de explotar la información cuantitativa que ofrece la Coordinadora, se realizaron diversas entrevistas focalizadas con representantes de la CONGDE. El análisis de datos secundarios reveló una serie de aspectos clave (tamaño, localización, tipo jurídico, profesionalización, base social, etc.) que sirvieron para confeccionar una variable compleja a partir de la cual se seleccionó una muestra de carácter intencional que reproducía la estructura del universo en relación con dichos aspectos.

Era necesario descender al plano individual y atender a cómo operan las dos racionalidades en las entidades y en los individuos concretos, puesto que, en definitiva, son las personas quienes hacen efectiva la institucionalización. Se llevó a cabo el trabajo de campo en 24 organizaciones y se analizaron cuatro tipos de información: material documental; 28 entrevistas semiestructuradas a directivos y trabajadores; datos del conjunto de la organización que sirvieron para aumentar la información en el nivel organizacional, y, finalmente, información cuantitativa y cualitativa del personal de la ONGD a través de una encuesta realizada a 99 individuos. Las organizaciones recogidas en la muestra constituyen el 22\% del total de la CONGDE y el 29\% de las que tienen su sede central en Madrid (que son el $70 \%$ de la Coordinadora). Por su parte, el personal entrevistado supone el $13 \%$ del total que poseen las ONGD en sus sedes centrales (el 63\% del personal de las ONGD que cuentan con 10 trabajadores o menos y el $26 \%$ del de las que poseen entre 11 y 30 ).

\section{Resultados de la investigación}

\section{A. La modificación del entramado institucional}

Según la teoría neoinstitucionalista, es difícil que suceda un cambio institucional. No obstante, puede ocurrir si sucede una variación del marco de relacio- 
nes de las organizaciones que operan en el mismo entorno. Pues bien, en España, se cuadruplicaron las ONGD en diez años. Se pasó de un entorno ciertamente estable, $y$ hasta cierto punto protegido para las ONGD de familia religiosa ${ }^{1}$, a otro mucho más incierto, donde surgían con fuerza organizaciones secularizadas; de una captación de fondos en parroquias, a una presencia cada vez mayor en los medios de comunicación. Todos estos cambios implicaron «modificaciones en los costos de transacción, que debían ser subsanados a través de transformaciones en el entramado institucional» (Romero, 1999: 25).

Estas modificaciones fueron propiciadas por la actuación de los medios de comunicación. En efecto, se ha tratado de explicar el boom de las ONGD en función del cambio de actitud gubernamental hacia la cooperación no oficial (Álvarez de Mon, 1998), del nacimiento de la CONGDE, del ingreso en la CEE (Ortega Carpio, 1994), de la aparición del estado del bienestar, que supuso la liberación de tiempo libre susceptible de dedicarse al voluntariado, así como de una crisis de valores (Felipe y Rodríguez, 1995), del desencanto de los actores de la política formal y los sindicatos (Serrano, 2001)... Pero el análisis detallado de cuando cambia la tendencia de la natalidad organizacional, precisamente en 1985, revela que el factor desencadenante (más que el aumento de los fondos públicos, como se cree habitualmente) fue el tratamiento que los medios de comunicación hicieron de la catástrofe de Etiopía (Ferré, 1997). Es decir, cuando se dieron las condiciones estructurales sociales, políticas y organizativas adecuadas, los medios resultaron ser el detonante que activó en la sociedad la movilización necesaria para que se potenciara la solidaridad organizada. Y si los medios provocaron el nacimiento de muchas ONGD, también supusieron un condicionante importante para la actuación de éstas y,

1. La familia o cultura de ONGD es una tipología empleada por Ortega Carpio (1994) que agrupa a las organizaciones en función de los espacios sociales donde nacen y que informa acerca de su orientación ideológica. Así, las ONGD de familia religiosa serían aquellas creadas por órdenes o congregaciones religiosas, colegios de esta orientación, así como parroquias u otras instancias eclesiales; ejemplo de este tipo serían Justicia y Paz, Manos Unidas o Madreselva, por ejemplo (suponen un 38,6\% de las 87 que componen el universo). Las político-sindicales son las creadas por sindicatos (ISCOD-UGT, SOTERMUN, etc.) y las más o menos vinculadas a partidos políticos (Fundación Cánovas del Castillo o Solidaridad Internacional) y son un $12,6 \%$. Las «solidarias» hacen referencia a las que nacieron como consecuencia de la simpatía que despertó en parte de la sociedad la experiencia de procesos revolucionarios en América Latina durante los años ochenta (Acsur, ASA, etc.), constituyen un 20,7\% del total. Las llamadas "profesionales» lo son porque suelen ser producto de la cooperación internacional de colectivos profesionales (MSF, Ingenierías Sin Fronteras, etc.); son un 17,3\% del total estudiado. De menor presencia son las ONGD internacionales $(5,8 \%)$ y universitarias (4,8\%), sedes en España de las ONGD de implantación internacional (como Ayuda en Acción o Acción contra el Hambre) o creadas desde espacios universitarios (Solidarios para el Desarrollo o Mon 3). Ortega Carpio también habla de ONGD «oficiales», es decir, aquéllas que han sido creadas desde instancias públicas (Fundación CEAR) o en las que, de alguna manera, participa el estado en su gobierno (Cruz Roja), razón por la que nosotros no las hemos considerado. Para una mayor profundización sobre el momento en el que surgen y sus características, véase López Rey (2006: 96-144). También está publicado un análisis detallado de las relaciones entre las diferentes tipologías (López Rey, 2001). 
especialmente, para la introducción de la lógica mercantil en la cultura propia del sector.

Los medios de comunicación son empresas que dirigen su atención a aquellos sucesos que logran mayores impactos en las audiencias. Sin embargo, la estructura de oportunidad mediática se cierra ante los discursos que cuestionan radicalmente el orden mundial y el sistema económico que lo sustenta (Sampedro, Jerez y López Rey, 2002), que son precisamente los que constituyen la base ideológica y el principio de acción de las ONGD. Por esta razón, el tipo de seguimiento que los medios hicieron del fenómeno de la cooperación internacional, guiado por criterios puramente empresariales y no de información y formación de la opinión pública, influyó, a su vez y de manera decisiva, en los procesos organizativos de las ONGD. En muchas de estas entidades, la racionalidad instrumental de tipo económico ha sustituido a la expresiva, porque ésta impide, o al menos dificulta, la adecuación de las organizaciones a los criterios de mercado con los que operan los medios. La racionalidad económica orienta la labor de los departamentos de comunicación y prensa de las ONGD hacia tareas más propias de departamentos de marketing social (centrados en la captación de recursos económicos y en la promoción institucional) que a las de difundir entre la sociedad discursos generadores de marcos de referencia alternativos. Como afirma Erro, las ONG olvidan sus mensajes conflictivos y los sustituyen por mensajes promocionales (Erro, 2002).

La investigación demostró también que, ante el miedo de perder cuotas en el mercado de la solidaridad, las ONGD tratan de no diferenciarse unas de otras recurriendo a autodefiniciones vagas, imprecisas y ambiguas, en la línea de una comunicación más opaca y trivial detectada en las organizaciones que se ven a sí mismas compitiendo con otras en un mercado (Nos Aldás, 2003). Son pocas las entidades que se posicionan abiertamente en opciones ideológicas, culturales o que permitan su identificación con sectores sociales de vinculación que les puedan privar de base social económica (donantes) o restar imagen pública.

\section{B. Evidencias de la percolación cultural en el nivel macro}

En el nivel del universo organizacional (87 ONGD), hemos determinado la existencia de dos tipos de comportamiento asimilables al fenómeno de la percolación. Este comportamiento diferenciado lo percibimos atendiendo a tres variables: el tipo jurídico, el tipo de base social promovido y el nivel de profesionalización. En efecto, existen diferencias importantes de comportamiento entre las ONGD en función de que sean asociaciones o fundaciones. Las primeras poseen una naturaleza jurídica de tipo personal y priman la participación, lo que se refleja en estructuras de poder más horizontales; las segundas, de naturaleza patrimonial, priorizan la gestión y, para asegurar tanto ésta como el patrimonio mismo, poseen una estructura jerárquica de corte piramidal.

Por otro lado, las ONGD más profesionalizadas poseen una estructura de financiación que las hace más dependientes de los fondos públicos, aunque 
de ellos obtienen una gran cantidad de ingresos. Las ONGD menos profesionalizadas son más pobres, pero más independientes o, mejor dicho, dependientes de recursos que generan ellas mismas.

Finalmente, muchas ONGD del tipo asociación pueden tener una base social política, la que tiene la capacidad de participar en las decisiones (los socios) y otra de tipo económico (los colaboradores económicos o donantes). El análisis reveló que las ONGD del tipo asociación que priorizan la colaboración económica acaban funcionando como si fueran fundaciones y las que potencian la participación de los socios no se preocupan tanto por mejorar en lo relativo a los aspectos económicos. En trabajos anteriores profundizamos en estos temas y analizamos detalladamente cómo se relacionan variables medidas a nivel macro como la familia de ONGD, la antigüedad, la forma jurídica, el nivel de profesionalización, el tamaño y el tipo de bases sociales, entre otras (López Rey, 2001).

\section{El nivel de los individuos: factores ideológicos que dificultan la percolación}

En el cuestionario que se pasó al personal contratado y voluntario, aparecían una serie de preguntas sobre la relación existente entre las ONGD y las empresas, sobre el mercado y el sistema económico. Con algunas de ellas, se elaboró una escala de siete puntos donde cada extremo representaba un tipo de solidaridad. Con otras, se realizó un análisis cluster que generó dos grupos que se correspondieron con los resultados de la escala. Ambos grupos pueden identificarse en función de la lógica institucional asumida por los individuos. Caracterizando a cada grupo en función de los rasgos ideológico-culturales, el definido por la solidaridad expresiva posee creencias religiosas más fuertes, dice que su vida cotidiana está condicionada por Dios en mayor medida y asiste a la iglesia con bastante frecuencia, situándose en una escala ideológica en una izquierda pura y dura.

El colectivo que hace presente los valores y prácticas del mercado cree menos fuertemente, no concede a Dios la misma importancia y no asiste a la iglesia con la misma frecuencia. Su orientación ideológica también es menos extrema y se autoubica en el centro izquierda.

Para confirmar nuestra hipótesis de que la religión y la ideología política influyen en la adopción de una u otra lógica institucional, llevamos a cabo un análisis de regresión lineal multivariante, estableciendo como variable criterio el índice de rechazo-aceptación de los valores de mercado y como independientes incluimos las que consideramos significativas. Resultó un modelo explicativo en función de la frecuencia con la que se asiste a la iglesia y la ideología política ${ }^{2}$, explicando ambas la misma proporción de la aceptación o el rechazo de los valores del mercado $(0,157$ en la $R$ corregida para ideología polí-

2. El modelo resultó significativo con $F=15,112$ y sig. 000 con 2 grados de libertad y un error típico de 1,$72072 ; R=0,566 ; R^{2}=0,321$ y $R^{2}$ corregida de 0,3 . 
tica, que se demostró la primera explicativa y 0,300 para el conjunto). Creemos que, con este dato, se confirma plenamente la hipótesis de que la ideología política y las creencias religiosas son elementos resistentes a la percolación cultural de valores de mercado. El hecho de que la variable explicativa sea la frecuencia con la que se asiste a misa y no la «fortaleza» de las creencias o la importancia concedida a Dios, se explica porque donde la Iglesia católica se asegura la transmisión de su filosofía (con respecto al dinero, la riqueza, etc.) es a través de los mecanismos de adoctrinamiento que ha desarrollado a lo largo de su historia para limitar la influencia de otras lógicas institucionales diferentes a la suya, como la del mercado.

En la tabla 1, aparecen extractos de las entrevistas mantenidas con directivos de las ONGD y se muestran los dos discursos enfrentados con respecto a una serie de aspectos que constituyen la vida cotidiana de las ONGD.

\section{El nivel organizacional: factores materiales que condicionan la percolación}

Fuera ya de las conclusiones de la investigación relacionadas con las hipótesis, existía la necesidad de acudir de nuevo al plano organizacional ${ }^{3}$ para responder a la pregunta jes el conflicto institucional un debate puramente ideológico entre los partidarios del mercado y sus detractores o, por el contrario, hay elementos materiales que sustentan en realidad a alguno de los dos discursos? Para responder a esta cuestión, procedimos a relacionar la asunción de la lógica mercantil con la eficiencia, relación que se mostró estadísticamente significativa. En otras palabras, cuanto mayor es la asunción de la racionalidad mercantil en las organizaciones, mayores son los rendimientos económicos.

Ahora bien, existe otro factor organizativo que está relacionado con la posible asunción de una u otra lógica y que es relevante para lo que estamos exponiendo: el acceso a fuentes privilegiadas de ingresos provenientes de instituciones madre. Las ONGD que globalmente son definidos como de solidaridad expresiva, poseen colectivos sociales específicos de los que pueden obtener fondos, voluntarios, asesoría especializada en diversas cuestiones, información y/o otro tipo de beneficios de manera exclusiva y en condiciones de privilegio, y con los que se establece una estrecha vinculación, cuando no una relación de dependencia. Por el contrario, las ONGD que no cuentan con estos proveedores/patrocinadores deben asegurar la supervivencia de la entidad por sí mismas, por lo que las exigencias de la vida organizativa se hacen más apremiantes. Hay una serie de aspectos como el pago de alquileres, de asesorías, etc. que no pueden ser incluidos en las partidas de los proyectos de desarrollo, y por eso

3. Los datos que ofrecemos en este apartado no se refieren a lo que hemos definido como universo organizacional (las 87 ONGD de la Coordinadora), sino que proceden de las 15 organizaciones a las que pudimos extrapolar los datos obtenidos de nuestra encuesta para caracterizar a las ONGD en función de la lógica mayoritaria existente entre sus miembros. El reducido número de las organizaciones obliga a considerar estos datos como ilustrativos, más que como definitivos. 
Tabla 1. Diferente visión de la realidad de las ONGD en función de la lógica institucionalizada (transcripciones literales de las entrevistas mantenidas con directivos de las organizaciones estudiadas).

\section{ONGD percoladas por una lógica proveniente del mercado}

Valoración de los recursos - A lo mejor sí, a lo mejor el modelo de éxito es el modelo... ¿̨económico? Es que económicos realmente el modelo económico al final lo que hace es que llegues a gente, es dinero.

Entorno en el que operan - Hay muchas, quizás demasiadas, ya que pueden desorientar a la gente, desperdigar (número de ONGD) los fondos $y$, por todo esto, se pierden esfuerzos.

- Esto tiene que tener un freno [...] es imposible que siga creciendo, que se pueda manejar tanto dinero [...] Y en ese proceso de selección se van a ir las más pequeñas.

Competencia entre ONGD - Existe un gran sentido patrimonial sobre las contrapartes [...] y hay competitividad. - Hay un gran miedo en todas las ONG, no sólo en la nuestra, a compartir información interesante, que al final es en esto en lo que está la valía de la ONG y su valor de mercado también. [...] Compartir tan alegremente no es tan fácil. [...] Es un mercado, pues que..., es un mercado.

El personal

- Habría que tener unos recursos humanos con criterio empresarial. Yo lo siento mucho, pero nosotros no somos un asilo, somos una ONG y hay que trabajar con eficacia.

El desarrollo

— Yo creo que todos estamos más o menos en el mismo barco ¿no? Se trata de ayudar a la gente, de solucionar los problemas que tiene, de que ellos solucionen los problemas. [...] Yo creo que todas las organizaciones tienen el mismo concepto del desarrollo, se diferencian en los distintos proyectos y en los distintos campos de actuación.

\section{ONGD con lógica expresiva resistente a la percolación}

- Porque [...] una organización que sepa hacer puentes [...] Hacen 5, 15, o 15.000 puentes. Bien, ¿y qué? Si luego hacemos análisis y el empobrecimiento, la injusticia..., son los problemas que identificamos.

- Yo no estoy a favor de reducir las ONG a pocas macro, porque las macro se convierten también en macroproyectos. ¿Y quién hace macro con los últimos? ¿Quién hace el proyecto de la traída de aguas que cuesta un millón de pesetas?

- Pero en estos espacios [supuestamente] progresistas de las ONG se tienen unas reticencias... Hay unas concepciones de las ONG como si fueran competidoras..., y yo no soy competencia de nadie, y nadie es competencia mía.

— Rechazamos el término de "recursos humanos», porque un recurso es algo que se explota $[\ldots]$ es instrumentalizar a la persona.

- Hay dos filosofías claras: la filosofía de una concepción de la ONG con un criterio puramente empresarial, que consiste en que seamos una empresa que hacemos proyectos de desarrollo y, por lo mismo, el dinero siempre es bueno; y otra, que es la que yo comparto, en la que la ONG no es una mera entidad financiera. Esta concepción entiende que la ONG tiene propuestas alternativas de un mundo o de una sociedad diferentes.

Las ONGD, instrumento de cambio social u organización técnica de cooperación internacional
- Es imposible. Nosotros no somos agentes de cambio social, no creo que las ONG sean agentes de cambio social. Son agentes que contribuyen al «campo» social, pero [...] el protagonismo ha de ser siempre político.

- Nosotros ya estamos curados de espanto de cosas como que las ONG van a cambiar el mundo y no sé qué y no sé cuánto. No, no estamos por esa filosofía, por muy bonita que sea, aunque yo entiendo que eso tiene que estar ahí como idea. Que eso tiene su cierto impacto social. Pero yo no me lo creo, Ahora bien, :que [una ONGD] es un instrumento técnico de cooperación? Obviamente, lo es.
- Las ONG deberíamos ser órganos de portavoz y participación de la ciudadanía.

- El proyecto de transformación es ideológico: yo pienso seguir luchando, y lucharé hasta que me muera, por un estilo de vida distinto.

- Si yo tuviera que renunciar a algo, y ahí represento a mi organización, renunciaría a hacer proyectos concretos. Jamás renunciaría a hacer sensibilización social. 
la búsqueda de recursos económicos para la propia ONGD se convierte en objetivo prioritario (si bien no recogido en los estatutos normalmente). Objetivo que entronca más fácilmente con una racionalidad mercantilista que con una expresiva.

Podemos caracterizar a las ONGD percoladas por la lógica institucional del mercado como organizaciones más jóvenes que las que mantienen una resistencia a la percolación cultural (la media de nacimiento organizacional se sitúa en 1.985 frente a 1.980 de las otras), con el doble de socios (964 por $410)$ y 20 veces más de colaboradores económicos (10.093 por 489). Sus recursos humanos están mucho más profesionalizados, tanto en las oficinas centrales $(51 \%$ frente a $28 \%$ ) como en el total de la organización (43\% frente a $9 \%$ ). Son las organizaciones más grandes y que mayor cantidad de recursos captan, sufriendo una dependencia mayor de las fuentes públicas de financiación $(77,4 \%$ por $37,4 \%)$. La mayor parte de su gasto lo destinan a proyectos de desarrollo ( $82 \%$ de sus presupuestos anuales), trabajando en menor medida la educación para el desarrollo y la sensibilización (11\%) y la ayuda de emergencia y humanitaria $(2,3 \%)$.

Por su parte, las ONGD que mantienen una solidaridad de tipo expresivo son más pequeñas, tanto en lo relativo al personal como al número de delegaciones, más antiguas y están menos profesionalizadas. Obtienen más de la mitad de sus recursos a través de cuotas y donaciones y, al ser sus presupuestos mucho más modestos, los gastos de administración suponen una parte más importante que en las percoladas (20\% frente al 5\%). Pero esta misma razón ilustra mejor el esfuerzo que realizan en materia de inversión destinada a la educación para el desarrollo y la sensibilización (15\%).

\section{Conclusiones}

Los estudios sobre el tercer sector en España están en auge y comienzan a publicarse algunos que abordan su relación con otros espacios sociales (Navarro y Rodríguez, 2004) y también los aspectos culturales (Izquieta y Callejo, 2004; Picas, 2003). En este sentido, creemos que la influencia cultural del mercado en el tercer sector es un hecho característico de las sociedades capitalistas contemporáneas que debe ser tenido en cuenta. Para su análisis, proponemos el concepto de percolación por su operatividad, precisión y posibilidades de desarrollo teórico, aunque no hayamos podido valorarlo en este momento en toda su complejidad. En efecto, por las características y las limitaciones específicas de esta investigación, creemos que conviene profundizar en el problema, con objeto de identificar de manera más precisa las dimensiones del fenómeno y, de este modo, prever las consecuencias de una escorrentía cultural.

Es necesario realizar estudios que aporten una mayor información para casos concretos de organizaciones. Aportaciones que permitan elaborar tipos ideales que reflejen adecuadamente los rasgos culturales propios de cada racionalidad. Que den cuenta, en profundidad y con detalle, del conflicto institucional que ocurre en el seno de las entidades y que lo vinculen con la estructura 
de las mismas, así como con otros aspectos de la vida organizacional. De igual modo, se deben determinar las dimensiones de las dos racionalidades en el plano individual, de manera que se arroje luz sobre los diversos factores que influyen en la asunción de cualquiera de las dos lógicas y que complementen a los aquí señalados. Pero también es necesario estudiar el fenómeno en otros ámbitos, como el de las organizaciones de acción social, entidades ecologistas, plataformas de voluntariado, etc. que confirmen o rebatan la percolación y escorrentía cultural en todo el tercer sector.

\section{Referencias bibliográficas}

AlberT, Réka; BARABÁSI, Albert-Laszlo (2002). «Statistical Mechanics of Complex Networks». Reviews of Modern Physics, 74, p. 47-97.

Allaire, Yvan; FIRSIROTU, Mihaela E. (1992). «Teorías sobre la cultura organizacional». En: VVAA. Cultura organizacional: Aspectos teóricos, prácticos y metodológicos. Santa Fe de Bogotá: Fondo Editorial Legis, p. 4-37.

Álvarez de Mon, Santiago; Martin Cavanna, Javier; Martínez, Juan Luis (1998). El tercer sector: retos y propuestas para el próximo milenio. Madrid: Ministerio de Trabajo y Asuntos Sociales.

BAigeS, Siscu; Dusster, David y otros (1996). Las ONG de desarrollo en España. Dilemas de la cooperación. Barcelona: Flor del Viento.

CASTELLS, Manuel (1998). La era de la información, vol. 3. Madrid: Alianza.

CERnUdA, Pilar (2002). Solidaridad: No es humanitario todo lo que reluce. Madrid: Temas de Hoy. Planeta-De Agostini.

DECKER, Ethan; FREY, Tim (1996). «Percolation Theory». En: <http://algodones.unm. edu/-ehdecker/complexity/96fall/percol.html>.

DesRoche, Henry (1983). Pour un traité d'economie sociale. París: CIEM.

DONATI, Pierpaolo (1997). «El desarrollo de las organizaciones del Tercer Sector». Reis, 79, p. 113-141.

- (1978). Publico e privato, fine di un'alternativa? Bolonia: Capelli.

- (1991). Teoria relazionale della societa. Milán: Franco Angeli.

Douglas, J. (1983). Why Charity? The Case for a Third Sector. Londres-Nueva Delhi: Sage.

DougLAS, Mary (1986). How Institutions Think. Siracusa: Syracuse University Press. ERro SALA, Javier (2002). Comunicación, desarrollo y ONGD. Bilbao: Hegoa.

ETKIN, J.; SCHWARSTEIN, L. (1989). Identidad de las organizaciones. Invariancia y cambio. Buenos Aires: Paidós.

Felipe, Ana de; Rodríguez de Rivas, Lilo (1995). Guía de la solidaridad. Madrid: Temas de Hoy.

FERRÉ, Jean Luc (1997). La acción humanitaria. Madrid: Paradigma.

GINER, Salvador (1995). «El altruismo asociativo en la sociedad civil. A modo de prefacio», prólogo de FUNES RIVAS, María Jesús. La ilusión solidaria. Las organizaciones altruistas como actores sociales en los regimenes democráticos. Madrid: UNED, p. 13-26.

HABERMAS, Jurgen (1987). Teoría de la acción comunicativa. Madrid: Taurus.

HansmanN, Henry (1987). «Economic Theories on Non-Profit Organization». En: Powell, Walter (ed.). The Nonprofit Sector. A research Handbook. New Haven: Yale University Press, p. 27-42. 
HERRERA GÓMEZ, Manuel (1998). El tercer sector en los sistemas de bienestar. Valencia: Tirant lo Blanch.

IzQuietA, J. L.; CAllejo, J. J. (2004). «Las organizaciones de ayuda humanitaria y de cooperación al desarrollo. Cultura e identidad de las ONGD». Reis, 105, p. 195-216.

JAMES, E. (1990). «Economics Theories of the Nonprofit Sector: A Comparative Perspective». En: AnHeier, H. K.; Seibel, Wolfang (eds.). The Third Sector: Comparative Studies of Nonprofit Organizations. Nueva York: De Gruyter.

KrashinKY, M. (1986). «Transaction Costs and a Theory of Nonprofit Organization». En: ROSE-ACKERMAN, Susan. The Economics of Nonprofit Institution. Nueva York: Oxford University Press, p. 114-132.

LEVESQUe, B. (ed.) (1989). L'autre economie. Une economie alternative? Sillery. Press de l'Université du Quebec.

LÓPEZ REY, José A. (2001): Solidaridad y mercado ¿Hay dos culturas en las ONGD? A Coruña: Netbiblo.

- (2006). El tercer sector y el mercado: Conflictos institucionales en España. Madrid: Centro de Investigaciones Sociológicas.

MiCELI, Jorge (2006). «La ciencia de las redes». Redes: Revista hispana para el análisis de redes sociales, 10 . Consultado en: $<$ http://revista-redes.rediris.es $>$.

Navarro Yáñez, C. J.; Rodríguez García, María Jesús (2004). «Administración pública y tercer sector. Propuesta analítica y estudio del caso de Andalucía». Papers, 73, p. 105-125.

NorTH, Douglass C. (1990). Institutions, Institutional Change and Economic Performances. Cambridge: Cambridge University Press.

Nos AldÁs, Eloísa (2003). «Diversidad y comunicación. Las posibilidades silenciadas del lenguaje». Convergencia, 33, p. 45-66.

OfFe, Claus (1984). Contradictions of the Welfare State. Cambridge: MIT Press.

Orella, José Luis (2000). «Desarrollo histórico». En: RUIZ DE OlabuÉnAGA, José I. (dir.). El sector no lucrativo en España. Fundación BBV, p. 45-77.

OrTega CARPIO, Ma Luz (1994). Las ONGD y la crisis del desarrollo: Un análisis de la cooperación con Centroamérica. Madrid: IEPALA.

OrTMAnN, Andreas; SCHLESINGER, Mark (1997). «Trust, Repute and the Role of NonProfit Enterprise». Voluntas, 8/2, p. 97-119.

PICAS CONTRERAS, Joan (2003). «El significado de la solidaridad. Ayuda humanitaria y derecho de injerencia». Papers, 71, p. 66-76.

Powell, Walter; Di Maggio, Paul J. (1991). «Introduction». En: Powell, Walter; Di MAGGIO, Paul J. (ed.). The new institutionalism in organizational analysis. Chicago-Londres: Chicago University Press, p. 1-38.

- (eds.) (1991). The new Institutionalism in Organizational Analysis. Chicago-Londres: Chicago University Press.

RiLEY, Patricia (1983). «A Structurationist Account of Political Cultures». Administrative Science Quarterly, 28/3, p. 414-437.

ROMERO, Jorge J. (1999). «Estudio introductorio. Los nuevos institucionalismos: sus diferencias, sus cercanías». En: POWELL, Walter; Di MAGGIO, Paul J. El nuevo institucionalismo en el análisis organizacional. México: Fondo de Cultura Económica, p. 7-29.

Salamon, Lester M. (1987a). «Partners in Public Service: The Scope and the Theory of Government Nonprofit Sector Relations». En: Powell, Walter (ed.). The Nonprofit Sector: A Research Handbook. New Haven: Yale University Press, p. 99-117. 
- (1987b). «Of Market Failure, Voluntary Failure and Third Part Government: Toward a Theory of Government - Non Profit Relations in the Modern Welfare State». Journal of Voluntary Action Research, 16, p. 29-47.

- (1999). «The Nonprofit Sector at a Crossroads: The Case of America». Voluntas, $10 / 1$, p. 5-23.

Salamon, Lester M.; AnHeier, Helmut K. (1992a). «In Search of the Non-Profit Sector I: The Question of Definitions». Voluntas, 3/2, p. 125-151.

- (1992b). «In Search of the Non-Profit Sector II: The Problem of Classification». Voluntas, 3/3, p. 267-309.

- (1998). "Social Origins of Civil Socety: Explaining the Nonprofit Sector CrossNationally». Voluntas, 9/3, p. 249-260.

Sampedro Blanco, Víctor; Jerez, Ariel; López Rey, José A. (2002). «ONG medios de comunicación y visibilidad pública. La ciudadanía ante la mediatización de los mensajes sociales». En: Revilla Blanco, Marisa (ed.). Las ONG y la política. Madrid: Istmo.

SCHEIN, Edgar H. (1988). La cultura empresarial y el liderazgo. Madrid: Plaza y Janés.

SERRANO, Maite (2001). «Las ONG entre la empresa y el Estado: ¿Cambio o reproducción del sistema?». En: NIETO PEREIRA, Luis (coord.). Cooperación para el desarrollo y ONG: Una visión crítica. Madrid: La Catarata/IUDC, p. 141-169.

SJÖSTRAND, Sven-Erik (2000). «The Organization of Nonprofit Activities». Voluntas, $11 / 3$, p. 199-216.

SMIRCICH, Linda (1983). "Concepts of Culture and Organizational Analysis». Administrative Science Quarterly, 28/3, p. 339-358.

SOGGE, David; ZADEK, Simon (1998). «iLeyes del mercado?». En: SOGGE, David (ed.). Compasión y cálculo: Un análisis crítico de la cooperación no gubernamental al desarrollo. Barcelona: Icaria Antrazyt TNI, p. 103-152.

WatTs, Duncan J. (2003). Six Degrees:The Science of a Connected Age. Londres: Random House.

Weisbrod, Burton A. (ed.) (1998). To Profit or Not to Profit: The Commercial Transformation of the Non-Profit Sector. Cambridge: Cambridge University Press. 